\title{
Feasibility Study on Improving Microwave Stroke Detection using Metamaterials
}

\author{
Eleonora Razzicchia, Navid Ghavami, Syed Ahsan, Panagiotis Kosmas \\ Faculty of Natural and Mathematical Sciences \\ King's College London \\ Strand, London, WC2R 2LS, United Kingdom \\ eleonora.razzicchia@kcl.ac.uk
}

\begin{abstract}
This paper presents a feasibility study to enhance microwave imaging (MWI) for intracerebral haemorrhage (ICH) detection using an innovative metamaterial (MM) design. To this end, we simulate a radar-based approach in CST Microwave Studio and use a previously developed algorithm based on Huygens principle to produce images with and without the MM. Our results indicate that the proposed MM film can significantly improve target localization and decrease image artefacts.
\end{abstract}

\section{INTRODUCTION}

Brain stroke is one of the leading causes of death and disability worldwide, and it is associated with increased economic costs of treatment and post-stroke care [1]. Intra-cerebral haemorrhage (ICH) is the second most common subtype of stroke and occurs when a blood vessel bursts, resulting in bleeding inside the brain. Currently, ICH detection relies on imaging methods such as computed tomography (CT) and magnetic resonance imaging (MRI). These two technologies are characterized by high costs and fail to provide a real time, compact and portable system which could be easily used by paramedics [2]. As an alternative, microwave imaging (MWI) methods and techniques are drawing the interest of many groups worldwide thanks to their low-cost, non-ionizing and non-invasive characteristics [3]. Diagnostic approaches in microwave brain imaging include both microwave tomography (MWT) as well as radar techniques, which can provide faster image processing [4].

A valid MWI device must include tailored hardware characteristics. For instance, to achieve a system that is capable of detecting a bleeding inside the brain, compact antennas operating below $2 \mathrm{GHz}$ immersed into a matching medium are recommended due to the strong attenuation of the EM waves inside the human head [5]. In addition, MM structures can be used to couple the incident power into the brain tissue. To this end, our previous studies have shown that tailored MM designs might be a powerful advance towards the development of MWT scanners [6]. This paper examines the potential of improving the quality of the reconstructed images by integrating MM technology in our radar-based imaging scanner.

The remainder of the paper is structured as follow. Section II reviews the algorithm and simulation setup. Section III presents and discusses the reconstruction results, and finally, Section IV concludes the work.

\section{Methods}

\section{A. Imaging Algorithm}

The MWI Huygens principle based algorithm presented in this paper was first introduced in [7] and has previously shown promising results for different applications [8], [9]. This algorithm measures the external surface field of the object of study and back-propagates it (virtually) into the imaging domain. Thus, according to the Huygens principle method, the reconstructed $2 \mathrm{D}$ E-field at location $\rho$ within the object is:

$$
E_{\mathrm{HP}}(\rho, m, f)=\sum_{n=1}^{N} E_{n m}(f) G\left(k\left|\rho_{n}-\rho\right|\right)
$$

where $E_{n m}$ is the field received from transmitter $m$ at receiver $n, G\left(k\left|\rho_{n}-\rho\right|\right)$ is the Green function defined in [7] and $k$ is the medium's wave number at frequency $f$.

\section{B. Simulation Setup}

We tested our MM design using the radar-based brain imaging scanner design shown in Fig. 11 1 . This system has been modelled in CST Microwave Studio and consists of 12 antennas arranged uniformly inside an elliptical headband filled with a matching medium made of a $90 \%$ glycerol-water mixture. To test the system, we used CST's EN 50361 Specific Anthropomorphic Mannequin (SAM) Phantom head model. This model comprises a bone shell filled with an elliptical brain.

To generate the reconstruction images, we assigned to the bone region permittivity and conductivity values of 20 and $0.35 \mathrm{~S} / \mathrm{m}$, respectively, while for brain these values have been considered as 45.8 and $0.76 \mathrm{~S} / \mathrm{m}$, respectively. We refer to this configuration as the "no target" scenario. We have repeated the simulations in the presence of a blood-mimicking target ("with target" scenario). The target is a cylinder of $40 \mathrm{~mm}$ height and $40 \mathrm{~mm}$ diameter and has been placed in three different positions inside the brain volume. We assigned properties of blood to the aforementioned target from CST's material library.

A $5 \times 6 \mathrm{MM}$ film whose unit cell is shown in Fig. 1p was placed on each antenna, adjacent to the substrate. The proposed unit cell comprises a copper pattern, which is a variation of the Jerusalem Cross split-ring resonator (SRR), sandwiched in between two Rogers RO3010 TM substrates 
(thickness $=1.27 \mathrm{~mm}, \epsilon^{\prime}=10.2$ and $\tan \delta=0.0022$ ). The Sparameters were calculated from the simulations in the 0.5-2.5 $\mathrm{GHz}$ frequency range, and the reconstruction images with and without the MM were generated.

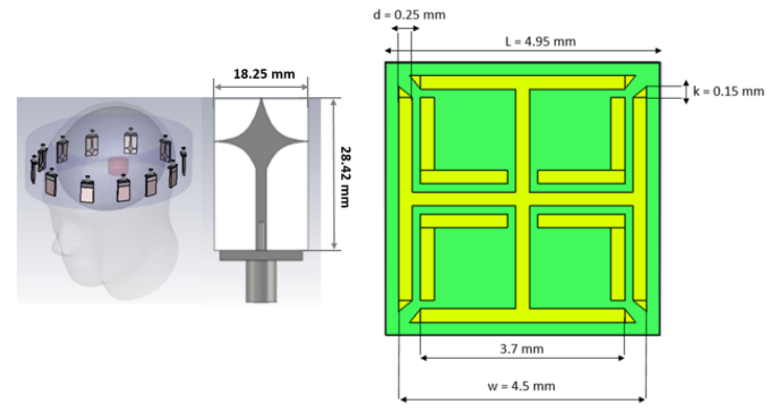

(a)

(b)

Fig. 1. (a) Radar-based microwave brain imaging scanner and spear-patch antenna [10], (b) MM unit cell geometry.

\section{RESULTS}

Fig. 2 presents the reconstructed normalized intensity images obtained for the domain inside the antenna array shown in Fig. 1. The blood-mimicking target has been reconstructed for three different positions, with and without the MM.
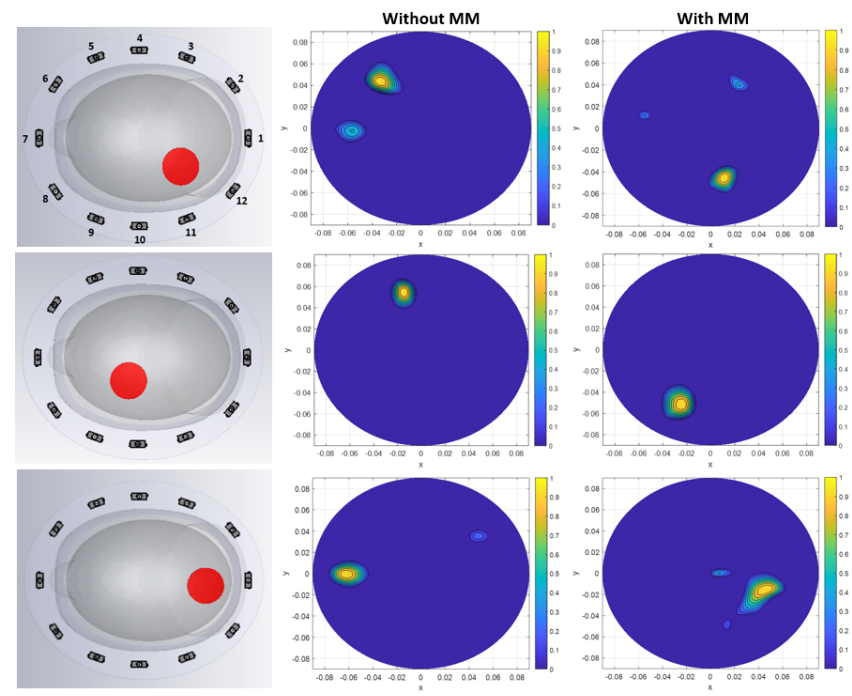

Fig. 2. Reconstructed images (with and without the MM) of the blood-like target positioned at three different locations inside the brain volume.

These results clearly indicate that the proposed MM design enables a correct detection and localization of the target and removes the noticeable imaging offset present when reconstructing the images without any MM. The target's identification is due to the presence of the MM, which can improve the antenna's return-loss and greatly enhance transmission, as shown in Fig. 3.

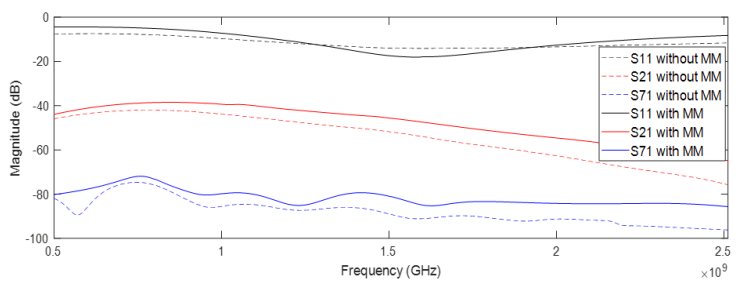

Fig. 3. S-Parameters showing an enhancement in the return-loss of Antenna 1. The transmission parameters $S_{71}$ and $S_{21}$ are also improved by about 7 $\mathrm{dB}$ at $1.5 \mathrm{GHz}$.

\section{CONCLUSIONS}

This study suggests that MM technology can improve ICH detection by enhancing signals from the target, which can then lead to more accurate detection with reduced image artefacts. Our ongoing work is focused on experimental studies to realistically assess the benefits from this approach.

\section{ACKNOWLEDGEMENT}

This research was supported in part by the EMERALD project funded from the European Union's Horizon 2020 under the Marie Skłodowska-Curie grant agreement No. 764479, and in part by UK's Engineering and Physical Sciences Research Council (EPSRC) under project grant EP/R013918/1.

\section{REFERENCES}

[1] S. Rajsic, H. Gothe, H. Borba, J. Vujicic, T. Toell, and U. Siebert, "Economic Burden of Stroke: A Systematic Review on Post-Stroke Care," Value Health, vol. 19, no. 7, p. A633, 2016.

[2] S. Mustafa, B. Mohammed, and A. Abbosh, "Novel preprocessing techniques for accurate microwave imaging of human brain," IEEE Antennas Wirel. Propag. Lett., vol. 12, pp. 460-463, 2013.

[3] M. Hopfer, R. Planas, A. Hamidipour, T. Henriksson, and S. Semenov, "Electromagnetic tomography for detection, differentiation, and monitoring of brain stroke: A virtual data and human head phantom study," IEEE Trans. Antennas Propag., vol. 59, no. 5, pp. 86-97, 2017.

[4] A. T. Mobashsher and A. M. Abbosh, "Developments of tomography and radar-based head imaging systems," in International Symposium on Antennas and Propagation (ISAP), Hobart, Tasmania, Australia, 2015.

[5] R. Scapaticci, L. Di Donato, I. Catapano, and L. Crocco, "A Feasibility Study on Microwave Imaging for Brain Stroke Monitoring," Prog. Electromagn. Res., vol. 40, no. January, pp. 305-324, 2014.

[6] E. Razzicchia, I. Sotiriou, H. Cano-Garcia, E. Kallos, G. Palikaras, and P. Kosmas, "Feasibility Study of Enhancing Microwave Brain Imaging Using Metamaterials," Sensors, vol. 19, no. 24, 2019.

[7] N. Ghavami, G. Tiberi, D. J. Edwards, and A. Monorchio, "UWB microwave imaging of objects with canonical shape," IEEE Trans. Anten. Propag. Propag., vol. 60, no. 1, pp. 231-239, 2012.

[8] N. Ghavami, G. Tiberi, M. Ghavami, S. Dudley, and M. Lane, "Huygens principle based UWB microwave imaging method for skin cancer detection," in International Symposium on Communication Systems, Networks and Digital Signal Processing (CSNDSP), Prague, Czech Republic, 2016.

[9] N. Ghavami, I. Sotiriou, and P. Kosmas, "Experimental Investigation of Microwave Imaging as Means to Assess Fruit Quality," in 13th European Conference on Antennas and Propagation, Krakow, Poland, 2019.

[10] G. Wei, S. Ahsan, and P. Kosmas, "Portable Microwave Imaging Head Scanners for Intracranial Haemorrhagic Detection," in Asia-Pacific Microwave Conference (APMC), Singapore, 2019. 